\title{
Physical exercise and dementia in patients with type 2 diabetes mellitus
}

\author{
Tomoyuki Kawada ${ }^{1}$
}

Received: 16 August 2016 / Accepted: 19 August 2016 / Published online: 7 September 2016

(C) Springer Science+Business Media New York 2016

I read with interest the recent review by Bertram et al. [1], in Endocrine. The authors overviewed the epidemiological association between type 2 diabetes mellitus (T2DM) and dementia, and speculated the biological mechanism on the association with special reference to chronic inflammation, oxidative stress, dyslipidemia, reduced $\beta$-amyloid clearance, and hormonal change. Thereafter, the authors intensively summarized the effect of physical exercise on dementia prevention in patients with T2DM. I fundamentally accept their recommendation of physical exercise, although biological evidence seems not enough to confirm the causal association. I have some concerns on their summary.

First, the authors summarized some intervention studies by using biomarkers. I recognize that each study keeps adequate design to know the positive (desirable) effects of training on the prevention of dementia. Unfortunately, T2DM progress varied in each study, and the prevalence of diabetic complication was not clearly described. T2DM is a chronic disease and it also relates to other metabolic components such as hypertension and dyslipidemia, which would be related to incident cardiovascular diseases. As T2DM is strongly associated with obesity via insulin resistance, caution should be paid to the change in obesity.

Second, there is a long-term process from sub-clinical cognitive impairment to clinical dementia. Namely, the association between T2DM and dementia should also be checked in pre-clinical stage of each disease. From the viewpoint of preventive strategy, inter-relationship between

Tomoyuki Kawada

kawada@nms.ac.jp

1 Department of Hygiene and Public Health, Nippon Medical School, 1-1-5 Sendagi, Bunkyo-Ku, Tokyo 113-8602, Japan cognitive impairment and impaired fasting glucose or impaired glucose tolerance seems important [2].

Finally, the authors did not discuss on sex difference and aging on the association [3]. As there are many factors affecting the association between T2DM and dementia $[4,5]$, net effect of physical exercise should be evaluated by further study.

\section{Compliance with ethical standards}

Conflict of interest The author declares that he has no conflict of interests.

\section{References}

1. S. Bertram, K. Brixius, C. Brinkmann, Exercise for the diabetic brain: how physical training may help prevent dementia and Alzheimer's disease in T2DM patients. Endocrine 53, 350-363 (2016)

2. L.D. Baker, L.L. Frank, K. Foster-Schubert, P.S. Green, C.W Wilkinson, A. McTiernan, B.A. Cholerton, S.R. Plymate, M.A. Fishel, G.S. Watson, G.E. Duncan, P.D. Mehta, S. Craft, Aerobic exercise improves cognition for older adults with glucose intolerance, a risk factor for Alzheimer's disease. J. Alzheimers Dis. 22, 569-579 (2010)

3. E.H. Mizrahi, A. Waitzman, T. Blumstein, M. Arad, A. Adunsky, Diabetes mellitus predicts cognitive impairment in patients with ischemic stroke. Am. J. Alzheimers Dis. Other Demen. 25, 362-366 (2010)

4. J.S. Roriz-Filho, T.M. Sá-Roriz, I. Rosset, A.L. Camozzato, A.C. Santos, M.L. Chaves, J.C. Moriguti, M. Roriz-Cruz, (Pre)diabetes, brain aging, and cognition. Biochim. Biophys. Acta. 1792, 432-443 (2009)

5. M. Baumgart, H.M. Snyder, M.C. Carrillo, S. Fazio, H. Kim, H. Johns, Summary of the evidence on modifiable risk factors for cognitive decline and dementia: A population-based perspective. Alzheimers Dement 11, 718-726 (2015) 